\title{
The Mass and Radius of the Neutron Star in EXO 1745-248
}

\author{
Feryal Özel ${ }^{1}$, Tolga Güver and Dimitrios Psaltis ${ }^{1}$ \\ University of Arizona, Department of Astronomy, 933 N. Cherry Ave., Tucson, AZ 85721
}

\begin{abstract}
Bursting X-ray binaries in globular clusters are ideal sources for measuring neutron star masses and radii, and hence, for determining the equation of state of cold, ultradense matter. We use time-resolved spectroscopic data from EXO 1745-248 during thermonuclear bursts that show strong evidence for photospheric radius expansion to measure the Eddington flux and the apparent surface area of the neutron star. We combine this with the recent measurement of the distance to the globular cluster Terzan 5, where this source resides, to measure the neutron star mass and radius. We find tightly constrained pairs of values for the mass and radius, which are centered around $M=1.4 M_{\odot}$ and $R=11 \mathrm{~km}$ or around $M=1.7 M_{\odot}$ and $R=9 \mathrm{~km}$. These values favor nucleonic equations of state with symmetry energy that is relatively low and has a weak dependence on density.
\end{abstract}

Subject headings: stars: neutron — X-rays: individual (EXO 1745-248)

\section{Introduction}

Accreting neutron stars that show thermonuclear X-ray bursts are optimal sources for determining the equation of state of cold, ultradense matter. They exhibit a number of spectroscopic phenomena that depend on the neutron star mass and radius, which can be used to measure these fundamental stellar properties. In particular, the apparent surface area of thermal emission during the cooling tail of a thermonuclear burst, as well as the peak flux achieved during strong, so-called photospheric radius expansion bursts, which reach the Eddington limit, provide two such observable quantities (van Paradijs 1978, 1979; see also Lewin, van Paradijs, \& Taam 1993).

The large collecting area and the systematic monitoring of a number of X-ray bursters by the Rossi X-ray Timing Explorer (RXTE) has generated a large database of high quality

\footnotetext{
${ }^{1}$ Steward Observatory, University of Arizona
} 
observations that we use in this study. The peak flux achieved in numerous photospheric radius expansion bursts from several sources has been shown to remain nearly constant, within a range as narrow as a few percent in 4U 1728-34 (Galloway et al. 2003), and the apparent surface areas to be reproducible between bursts (Galloway et al. 2008). This provides observational justification to the theoretical expectation that, in low magnetic field neutron stars, thermonuclear bursts not only quickly engulf the entire stellar surface but also that strong bursts reach an intrinsic limit associated with the Eddington luminosity.

These two spectroscopic phenomena can be combined either with another spectroscopic measurement, such as a surface redshift (Cottam, Paerels, \& Mendez 2002; see also Cottam et al. 2008) or with an accurate, independent distance to the neutron star, to break the intrinsic degeneracies in the neutron star properties and determine both the mass and the radius of the neutron star, independently (Özel 2006). X-ray bursters in globular clusters are unique in this respect, since the distances to the clusters can be independently measured. In this paper, we use the thermonuclear burst data of the source EXO 1745-248 located in Terzan 5 to measure the mass and radius of the neutron star.

The low mass X-ray binary EXO 1745-248 was discovered with Hakucho in August 1980 (Makishima et al. 1981). The source showed Type-I X-ray bursts, with intervals as short as $8 \mathrm{~min}$ (Inoue et al. 1984). It was again detected in 2000 during a RXTE/PCA scan of the galactic bulge as a transient X-ray burster (Markwardt \& Swank 2000). EXO 1745-248 was also observed by the Chandra X-ray Observatory in 2000 and 2003. Heinke et al. (2003) used the Chandra and RXTE observations to suggest that the source is an ultracompact binary and also identified a possible infrared counterpart in the Hubble Space Telescope (HST) images of the cluster. No burst oscillations have been reported from this source (Galloway et al. 2008).

Terzan 5 is one of the most metal rich globular cluster in the galaxy, with a metallicity close to solar (Origlia et al. 2004). Recently, Ortolani et al. (2007) revisited the distance measurements to Terzan 5 using HST/NICMOS data. Using NICMOS instrumental magnitudes and two separate reddening laws (Schlegel et al. 1998; Lee et al. 2001) to obtain the infrared extinction slope in the instrumental bands, they measured a distance of $6.3 \mathrm{kpc}$ to this cluster. The two main sources of error in this measurement are related to uncertainties in the color and magnitude measurements of the HB of the cluster, as well as to metallicity uncertainties, while the slope of the reddening law introduces a much smaller error. The combined error of $0.2 \mathrm{mag}$ corresponds to a distance uncertainty of about $10 \%$, which we will adopt here. Note that Ortolani et al. (2007) also used two calibrations for the conversion of the NICMOS to JHK magnitudes, which resulted in different values for the distance. Due to the significant width and the large displacement of the NICMOS F110W filter compared to 
the ground-based J filter, the transformations between these bands are color dependent and hence, suffer from systematic uncertainties that are difficult to quantify. For this reason, we will only use the distance measurement obtained from NICMOS instrumental magnitudes.

In this paper, we combine the distance measurement to Terzan 5 with the observations of radius expansion bursts obtained by RXTE to determine the mass and the radius of the neutron star in EXO 1745-248. In Section 2, we analyze the Eddington limited bursts from this source. In Section 3, we use these observations to determine the mass and radius of the neutron star and describe the formal method for assessing the uncertainties in the measurements using this technique. In Section 4, we discuss our results and compare them to several equations of state for neutron star matter.

\section{Spectral Analysis of X-ray Bursts}

EXO 1745-248 has been observed with RXTE for $148 \mathrm{ks}$, during which two Type-I Xray bursts were discovered, with clear evidence for photospheric radius expansion (Galloway et al. 2008). Note that, while Galloway et al. (2008) identified 20 more candidate non-PRE bursts that satisfied their trigger criteria, spectral analyses of those bursts revealed that the distinctive cooling associated with Type I bursts did not occur in these events, strongly suggesting that they are Type II instead (Lewin et al. 1993).

In order to analyze the PRE bursts, we extracted time resolved 2.5-25.0 keV X-ray spectra using the ftool seextrct and included the data from all the RXTE/PCA layers. We used the Science Event mode data with the E_125 $\mu$ s_64M_0_1s configuration, which has a nominal time resolution of $125 \mu \mathrm{s}$ in 64 spectral channels. We binned the X-ray spectra in 27 spectral channels and over $0.25 \mathrm{~s}$ (for count rates above $6000 \mathrm{ct} \mathrm{s}^{-1}$ ) and over $0.5 \mathrm{~s}$ (for count rates between 3000 and $6000 \mathrm{ct} \mathrm{s}^{-1}$ ) time intervals during each burst. Following Galloway et al. (2008), we extracted a 16 s spectrum prior to the burst and used it as background. We generated separate response matrix files for each burst using the PCARSP version 10.1 and took into account the offset pointing of the PCA during the creation of the response matrix files.

We fit the extracted spectra with a blackbody function, using the hydrogen column density value of $\mathrm{N}_{\mathrm{H}}=1.4 \times 10^{22} \mathrm{~cm}^{-2}$ determined by Wijnands et al. (2005) from Chandra observations. We used XSPEC v12 (Arnaud 1996) for our spectral analysis. For each spectrum, we calculated bolometric fluxes using equation (3) of Galloway et al. (2008). Figure 1 shows an example countrate spectrum as well as the best fit blackbody model. There are no systematic residuals in the fit, and the addition of any other spetcral components (e.g., 
a power-law model) is not statistically significant.

In Figure 2, we show the distribution of the $\chi^{2} /$ d.o.f. values that we obtained by fitting the X-ray spectra of the source during the 2 PRE bursts and compare it to the expected distribution for 25 degrees of freedom. All fits with $\chi^{2} /$ d.o.f. $<1.5$ follow the expected distribution and are, therefore, statistically acceptable. However, the five spectra with $\chi^{2} /$ d.o.f. $>1.5$ are outliers, which are likely to be dominated by systematic uncertainties. We rejected these fits from the subsequent analyses.

We show in Figure 3 the bolometric flux, the blackbody temperature, and the normalization of the model spectra during the evolution of the two PRE bursts. The characteristic decrease of the temperature and the increase of the photospheric radius around the burst peak, as well as the cooling of the burst emission at a constant photospheric radius for both bursts can be seen in both bursts.

In PRE bursts, the Eddington limit at the surface of the neutron star is thought to correspond to the point in each burst when the normalization of the blackbody gets its lowest value while the temperature reaches its highest (Damen et al. 1990). The spectral properties of the two PRE bursts during this so-called touchdown point are consistent with each other, as demonstrated in Figure 4. The combined best-fit value for the touchdown flux between the two bursts is $(6.25 \pm 0.20) \times 10^{-8} \mathrm{erg} \mathrm{cm}^{-2} \mathrm{~s}^{-1}$. Note that the ratios of the peak to touchdown fluxes in these two bursts are well within the value expected from the general relativistic effects alone, and therefore, this source is not subject to the bias discussed in Galloway, Özel, \& Psaltis (2008).

The second observational quantity that we determine from the spectral fits is the apparent radius of the emitting region during the cooling phase of the bursts. This is given directly by the normalization of the blackbody function, $A \equiv\left(R_{\mathrm{app}} / D\right)^{2}$, where $R_{\mathrm{app}}$ is the radius corresponding to the apparent emitting surface area and $D$ is the distance to the source. We chose the intervals $4.5-15 \mathrm{~s}$ in both bursts, during which the apparent radius is constant. Fitting the cooling tails of these bursts individually resulted in values for the ratio $A=104.0 \pm 1.0 \mathrm{~km}^{2} \mathrm{kpc}^{-2}$ and $A=130.0 \pm 1.0 \mathrm{~km}^{2} \mathrm{kpc}^{-2}$. Similar systematic uncertainties have been observed in the Eddington fluxes from PRE bursts from, e.g., 4U 1728-34 (Galloway et al. 2003) and have been attributed to the variable reflection off of the accretion disk that changes at a superorbital period. Such a phenomenon can introduce systematic uncertainties in the apparent surface areas measured during the cooling tails of bursts. Because the systematic errors dominate over the statistical errors in this particular case, we will assume a boxcar probability distribution over this quantity in the range $A=116 \pm 13 \mathrm{~km}^{2} \mathrm{kpc}^{-2}$. 


\section{Determination of the Neutron Star Mass and Radius}

In an approach similar to Özel (2006), we use the spectroscopic measurements of the touchdown flux $F_{\mathrm{TD}}$ and the ratio $A$ during the cooling tails of the bursts, together with the measurement of the distance $D$ to the source in order to determine the neutron star mass $M$ and radius $R$. The observed spectroscopic quantities depend on the stellar parameters according to the relations

$$
F_{\mathrm{TD}}=\frac{G M c}{k_{\mathrm{es}} D^{2}}\left(1-\frac{2 G M}{R c^{2}}\right)^{1 / 2}
$$

and

$$
A=\frac{R^{2}}{D^{2} f_{\mathrm{c}}^{4}}\left(1-\frac{2 G M}{R c^{2}}\right)^{-1},
$$

where $G$ is the gravitational constant, $c$ is the speed of light, $k_{\text {es }}$ is the opacity to electron scattering, and $f_{\mathrm{c}}$ is the color correction factor.

In the absence of errors in the determination of the observable quantities, the last two equations can be solved for the mass and radius of the neutron star. However, because of the particular dependences of $F_{\mathrm{TD}}$ and $A$ on the neutron star mass and radius (see also Fig. 1 in Özel 2006), the loci of mass-radius points that correspond to each observable intersect, in general, at two distinct positions. Moreover, the diverse nature of uncertainties associated to each of the observables requires a formal assessment of the propagation of errors, which we present here.

We assign a probability distribution function to each of the observable quantities and denote them by $P(D) d D, P\left(F_{\mathrm{TD}}\right) d F_{\mathrm{TD}}$, and $P(A) d A$. Because the various measurements that lead to the determination of the three observables are independent of each other, the total probability density is simply given by the product

$$
\begin{aligned}
& P\left(D, F_{\mathrm{TD}}, A\right) d D d F_{\mathrm{TD}} d A= \\
& P(D) P\left(F_{\mathrm{TD}}\right) P(A) d D d F_{\mathrm{TD}} d A .
\end{aligned}
$$

Our goal is to convert this probability density into one over the neutron-star mass, $M$, and radius, $R$. We will achieve this by making a change of variables from the pair $\left(F_{\mathrm{TD}}, A\right)$ to $(M, R)$ and then by marginalizing over distance. Formally, this implies that

$$
\begin{gathered}
P(D, M, R) d D d M d R=\frac{1}{2} P(D) P\left[F_{\mathrm{TD}}(M, R, D)\right] \\
P[A(M, R, D)] J\left(\frac{F_{\mathrm{TD}}, A}{M, R}\right) d D d M d R
\end{gathered}
$$


where $J\left(F_{\mathrm{TD}}, A / M, R\right)$ is the Jacobian of the transformation. It is important to emphasize here that, given a distance $\mathrm{D}$, not all pairs of the observables $\left(F_{\mathrm{TD}}, A\right)$ can be obtained with real values for the neutron-star mass and radius. For this reason, the final distribution will not be normalized, even if the three distributions of equation (3) are. In addition, the factor $1 / 2$ appears in equation (4) because nearly all pairs of the observables $\left(F_{\mathrm{TD}}, A\right)$ correspond to two distinct pairs of $(M, D)$. There is only a region of the parameter space for which the pair of observables corresponds to a single pair of values for the mass and radius. However, this region has zero volume and, therefore, will not contribute to the final probability distribution after we marginalize over distance.

We can now use the above expressions to calculate the Jacobian of the transformation

$$
\begin{aligned}
J\left(\frac{F_{\mathrm{TD}}, A}{M, R}\right)= & \frac{G c R}{f_{\mathrm{c}}^{4} k_{\mathrm{es}} D^{4}}\left[1-\frac{6 G M}{R c^{2}}+7\left(\frac{G M}{R c^{2}}\right)\right] \\
& \left(1-\frac{2 G M}{R c^{2}}\right)^{-5 / 2} .
\end{aligned}
$$

For the source EXO 1745-248, the distance measurement is dominated by systematic errors as discussed in $\S 1$. We will, therefore, use a box-car probability distribution over distance, with a mean of $D_{0}=6.3 \mathrm{kpc}$ and a range of $\Delta D=0.1 D_{0}$, i.e.,

$$
P(D) d D= \begin{cases}\frac{1}{\Delta D} & \text { if }\left|D-D_{0}\right| \leq \Delta D / 2 \\ 0 & \text { otherwise }\end{cases}
$$

The measurements of the touchdown flux is consistent between the two radius-expansion bursts (see Fig. 4) and is, therefore, dominated only by statistical uncertainties. We assign a Gaussian probability distribution for this quantity with a mean and a standard deviation that we estimate by fitting a Gaussian function to the product of the probability distributions that correspond to the confidence contours shown in Figure 4. The result is a mean of

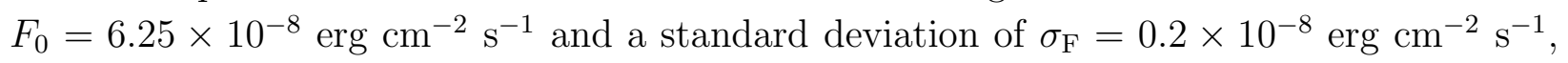
i.e.,

$$
P\left(F_{\mathrm{TD}}\right) d F_{\mathrm{TD}}=\frac{1}{\sqrt{2 \pi \sigma_{\mathrm{F}}^{2}}} \exp \left[-\frac{\left(F_{\mathrm{TD}}-F_{0}\right)^{2}}{2 \sigma_{\mathrm{F}}^{2}}\right] .
$$

Finally, the measurement of the ratio $A \equiv\left(R_{\text {app }} / D\right)^{2}$ between the two bursts is dominated by systematic uncertainties. We, therefore, assign to this ratio a box car probability distribution with a mean of $A_{0}=116$ and a range of $\Delta A=26$, i.e.,

$$
P(A) d A= \begin{cases}\frac{1}{\Delta A} & \text { if }\left|A-A_{0}\right| \leq \Delta A / 2 \\ 0 & \text { otherwise }\end{cases}
$$


The color correction factor that enters these expressions is determined by models of burning neutron star atmospheres (e.g., Madej et al. 2004). At the observed high temperatures of the bursts, as well as in the absence of significant magnetic fields or heavy elements (as evidenced by the lack of atomic transition lines in the high resolution spectra), the Comptonized radiative equilibrium atmosphere models can be reliably calculated. As discussed in Özel (2006), when the emerging flux is substantially sub-Eddington, as in the case of the cooling tails of the bursts, the color correction factor $f_{\infty}$ asymptotes to a value of $\simeq 1.4$, which we adopt here. Finally, we use the electron scattering opacity $\kappa_{e s}=0.20(1+X) \mathrm{cm}^{2} \mathrm{~g}^{-1}$, that depends on the hydrogen mass fraction $X$.

We obtain the final distribution over neutron-star mass and radius by inserting equations (5)-(8) into equation (44) and integrating over distance. Figure 5 shows the 1 - and $2-\sigma$ contours for the mass and radius of the neutron star in EXO 1745-248, for a hydrogen mass fraction $X=0$. For larger values of the hydrogen mass fraction $(X \gtrsim 0.1)$, the masses and radii inferred individually from the Eddington limit and the apparent surface area become rapidly inconsistent with each other. This result is in line with the identification of EXO 1745-248 with an ultracompact binary by Heinke et al. (2003). Note that there are two distinct regions in the mass-radius plane that are consistent with the data because of the particular dependence of $F_{\mathrm{TD}}$ and $A$ on the stellar mass and radius, as discussed above.

\section{Discussion}

We used time-resolved spectroscopic data from EXO 1745-248 during thermonuclear bursts that show strong evidence for photospheric radius expansion to measure the Eddington flux and the apparent surface area of the neutron star. We combined this with the recent measurement of the distance to the globular cluster Terzan 5 (Ortolani et al. 2007), where this source resides, to measure the neutron star mass and radius. We found tightly constrained pairs of values for the mass and radius, which are centered around $M=1.4 M_{\odot}$ and $R=$ $11 \mathrm{~km}$ or around $M=1.7 M_{\odot}$ and $R=9 \mathrm{~km}$.

The confidence contours on the mass-radius plane (see Fig. 5) are in best agreement with nucleonic equations of state without the presence of condensates or strange matter. The leftmost family of mass-radius relations is based on the assumption that the absolute ground state of matter is made up of an approximately equal mixture of up, down, and strange quarks. The primary difference between the other two families of mass-radius relations is the symmetry properties of the equation of state of neutron star matter. Moreover, the mass-radius relations with deflection points are characteristic of calculations that incorporate bosons that can condense and, thus, soften the equation of state at high densities. The radius 
measurements presented here favor relatively low values for the bulk symmetry energy with a weak density dependence (see Lattimer \& Prakash 2001).

The measurement of the mass and radius of a neutron star can significantly constrain the range of possibilities for the equation of state of ultradense matter, as discussed above. However, it cannot uniquely pinpoint to a single equation of state because of both the measurement errors and the uncertainties in the fundamental parameters that enter the nuclear physics calculations, such as the symmetry energy of nucleonic matter or the bag constant for strange stars. Further, even tighter constraints on the equation of state can be obtained by combining observations of neutron stars with different masses that will distinguish between the slopes of the predicted mass-radius relations, which are determined entirely by the physics of the neutron star interior.

A number of other constraints on neutron star radii have been obtained to date using various methods. Özel (2006) used spectroscopic measurements of the Eddington limit and apparent surface area during thermonuclear bursts, in conjuction with the detection of a redshifted atomic line from the source EXO 0748-676, to determine a mass of $M \geq 2.10 \pm$ $0.28 M_{\odot}$ and a radius $R \geq 13.8 \pm 1.8 \mathrm{~km}$. This radius measurement is consistent with the one presented in the current paper to within $2-\sigma$, and, therefore, several nucleonic equations of state are consistent with both measurements.

Radii have also been measured from globular cluster neutron stars in binaries emitting thermally during quiescence, such as X7 in 47 Tuc and others in $\omega$ Cen, M 13, and NGC 2808 (Heinke et al. 2006; Webb \& Barret 2007). (Note that we do not consider here isolated neutron stars such as RX J1856-3754 because of the unquantified systematic uncertainties arising from the apparent temperature anisotropies on the neutron star surfaces and their probable magnetic nature; see Walter \& Lattimer 2002; Braje \& Romani 2002; Tiengo \& Mereghetti 2007). These measurements have carved out large allowed bands in the massradius plane, all of which are also consistent with equations of state that predict neutron stars with radii $R \sim 11 \mathrm{~km}$. Future tight constraints on the masses and radii of additional neutron stars with these and other methods (see, e.g., Lattimer \& Prakash 2007) will resolve this long-standing question of high energy astrophysics.

We thank Rodger Thompson for his help with understanding the NICMOS calibrations, Duncan Galloway for his help with burst analyses, Adrienne Juett for bringing the source to our attention, Jim Lattimer for sharing with us the mass-radius relations, and Martin Elvis for useful conversations on constraining the neutron star equation of state. We also thank an anonymous referee for useful suggestions. F. Ö. acknowledges support from NSF grant AST 07-08640. D. P. is supported by the NSF CAREER award NSF 0746549. 


\section{REFERENCES}

Arnaud, K. A. 1996, Astronomical Data Analysis Software and Systems V, 101, 17

Braje, T. M., \& Romani, R. W. 2002, ApJ, 580, 1043

Cottam, J., Paerels, F., \& Mendez, M. 2002, Nature, 420, 51

Cottam, J., Paerels, F., Méndez, M., Boirin, L., Lewin, W. H. G., Kuulkers, E., \& Miller, J. M. 2008, ApJ, 672, 504

Damen, E., Magnier, E., Lewin, W. H. G., Tan, J., Penninx, W., \& van Paradijs, J. 1990, A\&A, 237, 103

Galloway, D. K., Psaltis, D., Chakrabarty, D., \& Muno, M. P. 2003, ApJ, 590, 999

Galloway, D. K., Muno, M. P., Hartman, J. M., Psaltis, D., \& Chakrabarty, D. 2006, ApJ, in press arXiv:astro-ph/0608259)

Galloway, D. K., Özel, F., \& Psaltis, D. 2008, MNRAS, 387, 268

Heinke, C. O., Edmonds, P. D., Grindlay, J. E., Lloyd, D. A., Cohn, H. N., \& Lugger, P. M. 2003, ApJ, 590, 809

Heinke, C. O., Rybicki, G. B., Narayan, R., \& Grindlay, J. E. 2006, ApJ, 644, 1090

Inoue, H., et al. 1984, PASJ, 36, 855

Lattimer, J. M., \& Prakash, M. 2001, ApJ, 550, 426

Lattimer, J. M., \& Prakash, M. 2007, Phys. Rep., 442, 109

Lee, J.-W., Carney, B. W., Fullton, L. K., \& Stetson, P. B. 2001, AJ, 122, 3136

Lewin, W. H. G., van Paradijs, J., \& Taam, R. E. 1993, Space Science Reviews, 62, 223

Madej, J., Joss, P. C., Rózańska, A. 2004, ApJ, 602, 904

Makishima, K., et al. 1981, ApJ, 247, L23

Markwardt, C. B., \& Swank, J. H. 2000, IAU Circ., 7454, 1

Origlia, L., \& Rich, R. M. 2004, AJ, 127, 3422

Ortolani, S., Barbuy, B., Bica, E., Zoccali, M., \& Renzini, A. 2007, A\&A, 470, 1043 
Özel, F. 2006, Nature, 441, 1115

Schlegel, D. J., Finkbeiner, D. P., \& Davis, M. 1998, ApJ, 500, 525

Tiengo, A., \& Mereghetti, S. 2007, ApJ, 657, L101

van Paradijs, J. 1978, Nature, 274, 650

van Paradijs, J. 1979, ApJ, 234, 609

Walter, F. M., \& Lattimer, J. M. 2002, ApJ, 576, L145

Webb, N. A., \& Barret, D. 2007, ApJ, 671, 727

Wijnands, R., Heinke, C. O., Pooley, D., Edmonds, P. D., Lewin, W. H. G., Grindlay, J. E., Jonker, P. G., \& Miller, J. M. 2005, ApJ, 618, 883 


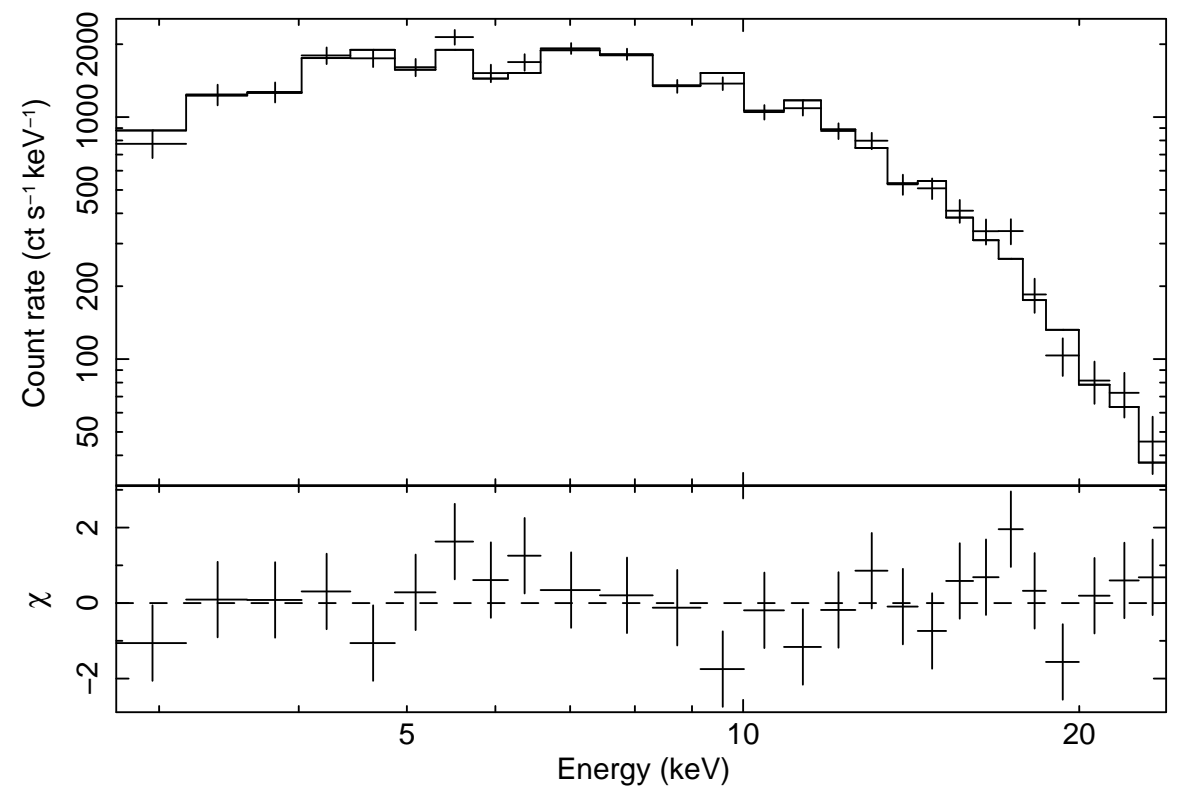

Fig. 1.- An example count rate spectrum of EXO 1745-248 together with the best-fit blackbody model. The lower panel shows the residuals of the fit, defined as $\chi=\left(x_{i}-x_{m}\right) / \sigma_{i}$, where $x_{i}$ and $\sigma_{i}$ are the observed counts and uncertainty, respectively, in the $\mathrm{i}$-th spectral bin, and $x_{m}$ is the model prediction. This example corresponds to the touchdown point of the burst shown in the left panel of Figure 3. 


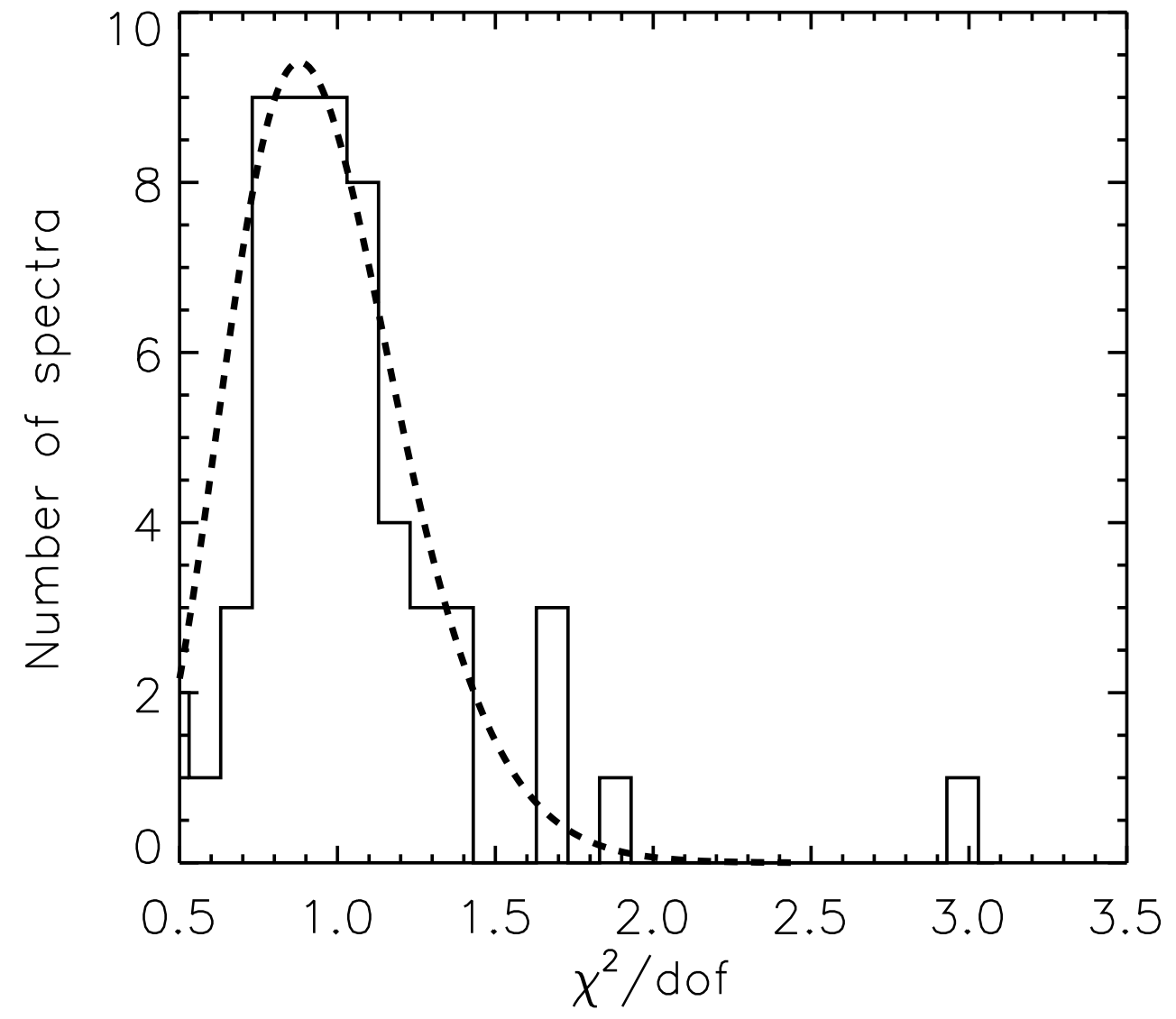

Fig. 2.- The histogram shows the distribution of $\chi^{2} /$ d.o.f. values obtained by fitting the spectra during the evolution of the two photospheric radius bursts of EXO 1745-248. the dashed line shows the expected $\chi^{2} /$ d.o.f. distribution for 25 degrees of freedom. The five spectral fits with $\chi^{2} /$ d.o.f. $>1.5$ are outliers and are excluded from the subsequent analyses. 

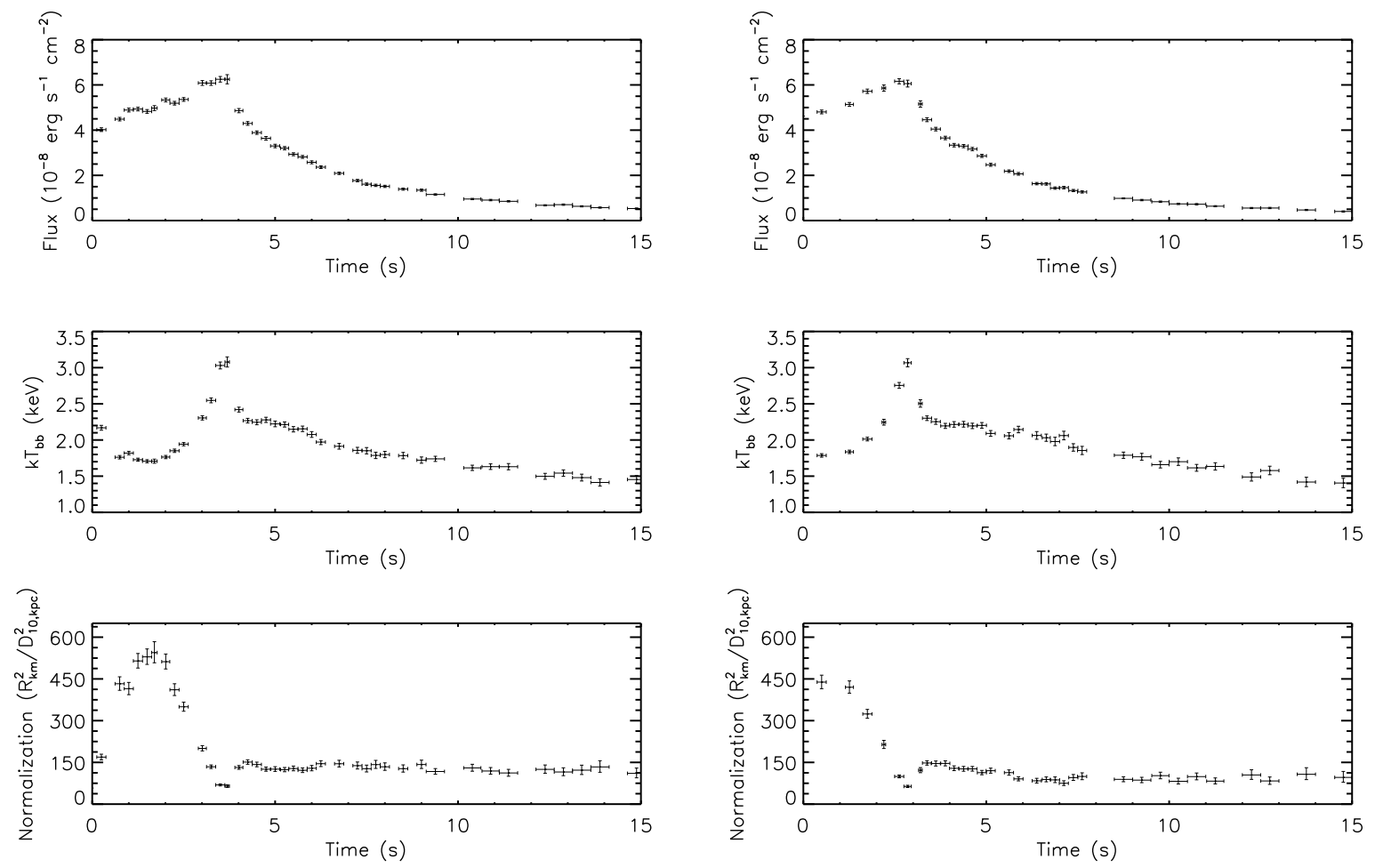

Fig. 3.- The spectral evolution during the first 15 seconds of the two Eddington limited thermonuclear bursts observed from EXO 1745-248 by RXTE. The panels show the evolution of the flux, the blackbody temperature and the apparent radius as observed at infinity, together with their $1-\sigma$ statistical errors. 


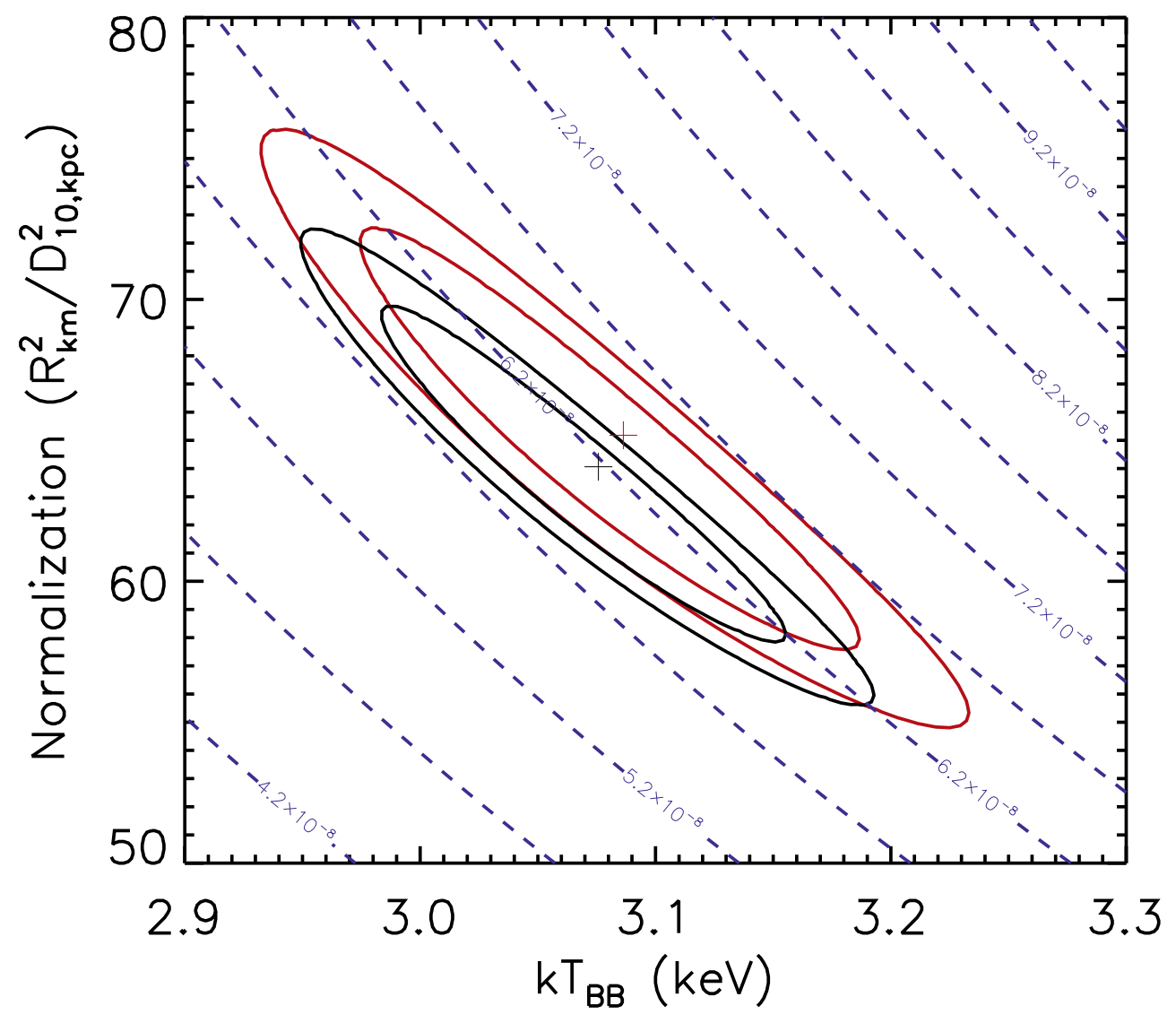

Fig. 4.- The 1- and 2- $\sigma$ confidence contours of the normalization and blackbody temperature obtained from fitting the two PRE bursts during touchdown. The dashed lines show contours of constant bolometric flux. 


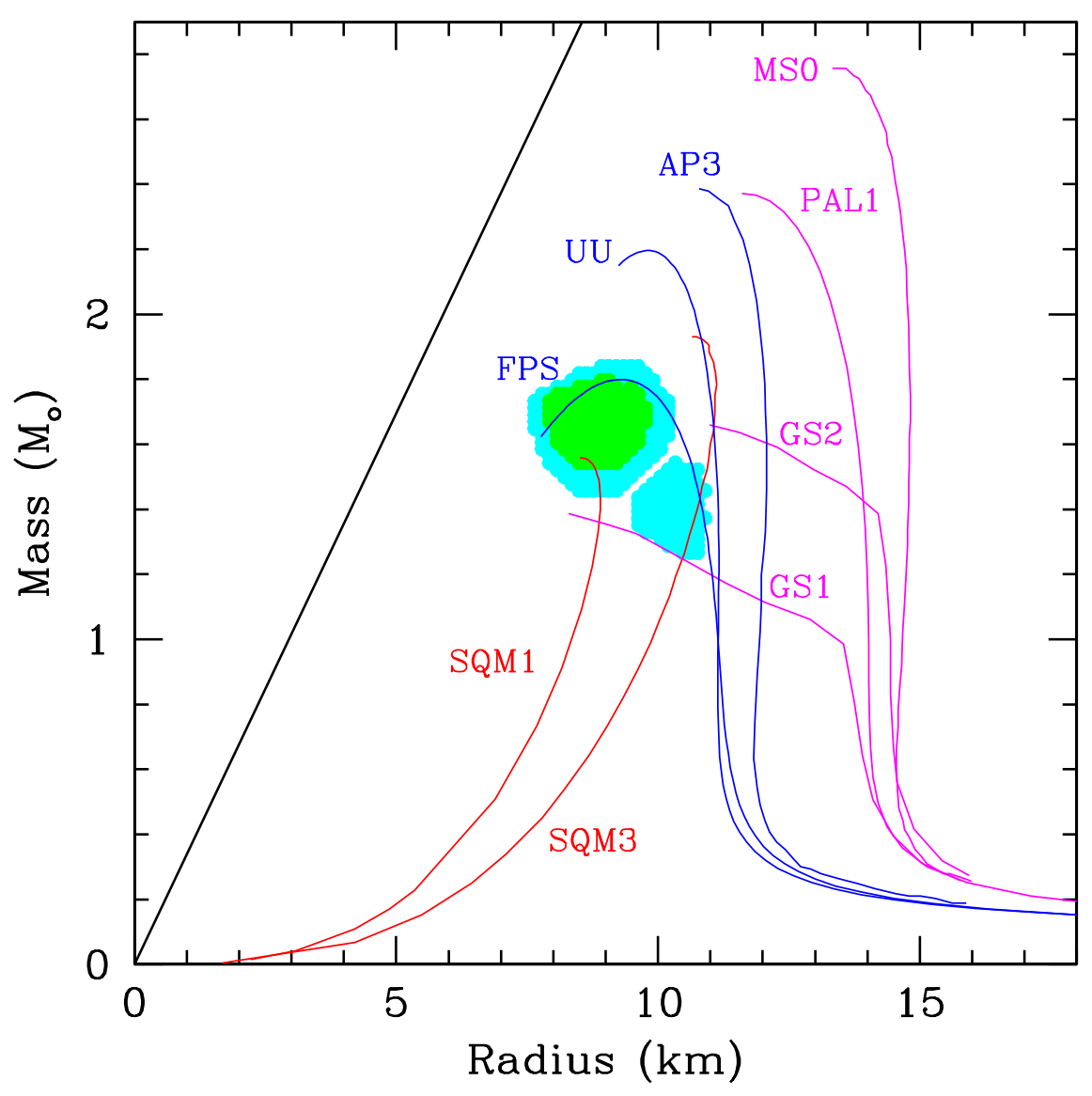

Fig. 5.- The $1-$ and $2-\sigma$ contours for the mass and radius of the neutron star in EXO $1745-248$, for a hydrogen mass fraction of $X=0$, based on the spectroscopic data during thermonuclear bursts combined with a distance measurement to the globular cluster. Neutron star radii larger than $\sim 13 \mathrm{~km}$ are inconsistent with the data. The descriptions of the various equations of state and the corresponding labels can be found in Lattimer \& Prakash (2001). 